IDEAS IN ECLLGGY AND EVRLuTION 5: 9-12, 2012

doi:10.4033/iee.2012.5.3.e

(C) 2012 The Author. (C) Ideas in Ecology and Evolution 2012

Received 3 May 2012; Accepted 3 May 2012

\title{
Editarial
}

\section{Are peer-review filters optimal for the progress of science in ecology and evolution?}

\author{
Lonnie W. Aarssen \\ Lonnie W. Aarssen (aarssenl@queensu.ca), Department of Biology, Queen's University, Kingston, ON, Canada \\ KTL $3 N 6$
}

The progress of science depends on dissemination of discovery. This is achieved through publication, and most effectively when filtered through peer review. Peer-review filters typically involve expert judgments concerning several manuscript features, such as the clarity of objectives/hypotheses, effectiveness of methodology, efficacy of data analyses, quality of data presentation, completeness of literature citation, justification for interpretations/conclusions, quality of English and writing style, novelty, and potentials for impact on theory, informing application, and inspiring future research. Pre-publication peer-review filters therefore help to ensure that reports of discoveries are as useful to readers as possible because assessment and constructive feedback from peers regarding the above features usually improves the overall quality of manuscripts. This in turn generates a body of published literature through which readers can navigate with relative ease and confidence to learn of those discoveries that are most useful for the progress of their own research interests, or for addressing the needs of society.

But how rigorous should peer-review filters be? Is the current practice optimal for the progress of science-particularly in ecology and evolution? A simple optimality model can be used to generate needed conversation for these important questions. The rigor of peer-review filters can range from zero, i.e. publish without peer review, to the highest level where publication is granted for 'only the best of the best', as judged by peer review (Figure 1). As the number and rigor of peer-review filters increases from zero, benefits can be expected to accrue rapidly but with a decelerating rate toward a plateau.

However, this does not mean that 'more is better'. Excessively stringent peer-review standards impose significant costs on the progress of science that accelerate rapidly toward the highest end of the scale (Figure 1). Specifically, publishing 'only the best of the best' can leave meritorious papers unpublished and hence unavailable to science. It also amounts to censorship, leaving authors marginalized, not unlike those who were at one time required to sit at the back of the bus because of their skin colour, or were unsuccessful in job applications because of their gender. When told that their manuscripts are good, but not good enough for publication in the editor's journal-nor can they be made good enough with revision-researchers can feel unfairly treated and despondent. They become susceptible to retaliatory response, eventually manifesting as habitual bias in their own reviewing behaviours. And this bias is hard to expose because reviewers can hide behind anonymity with the traditional single-blind peer-review policy still espoused by virtually all journals in ecology and evolution. There are also no effective deterrents against reviewer bias because, with no remuneration/ compensation, reviewers are not competing with each other for opportunities to review. In order to maximize their profits, publishers/editors need reviewers that are willing to provide their services for free, and so membership in the reviewer 'pool' cannot be strictly policed. The end result is widespread erosion of reviewer incentive, and of those reviews that editors manage to get, many are arbitrary, or poor in quality. These costs of anonymous peer review far outweigh any supposed benefits of anonymity.

Draconian peer-review standards are a product of gate-keeping elitism, motivated by self-serving goals of journal publishers and editors to elevate impact factor as a symbol of status, and to compete with other journals for that status. In return, publishers get elevated profits, 


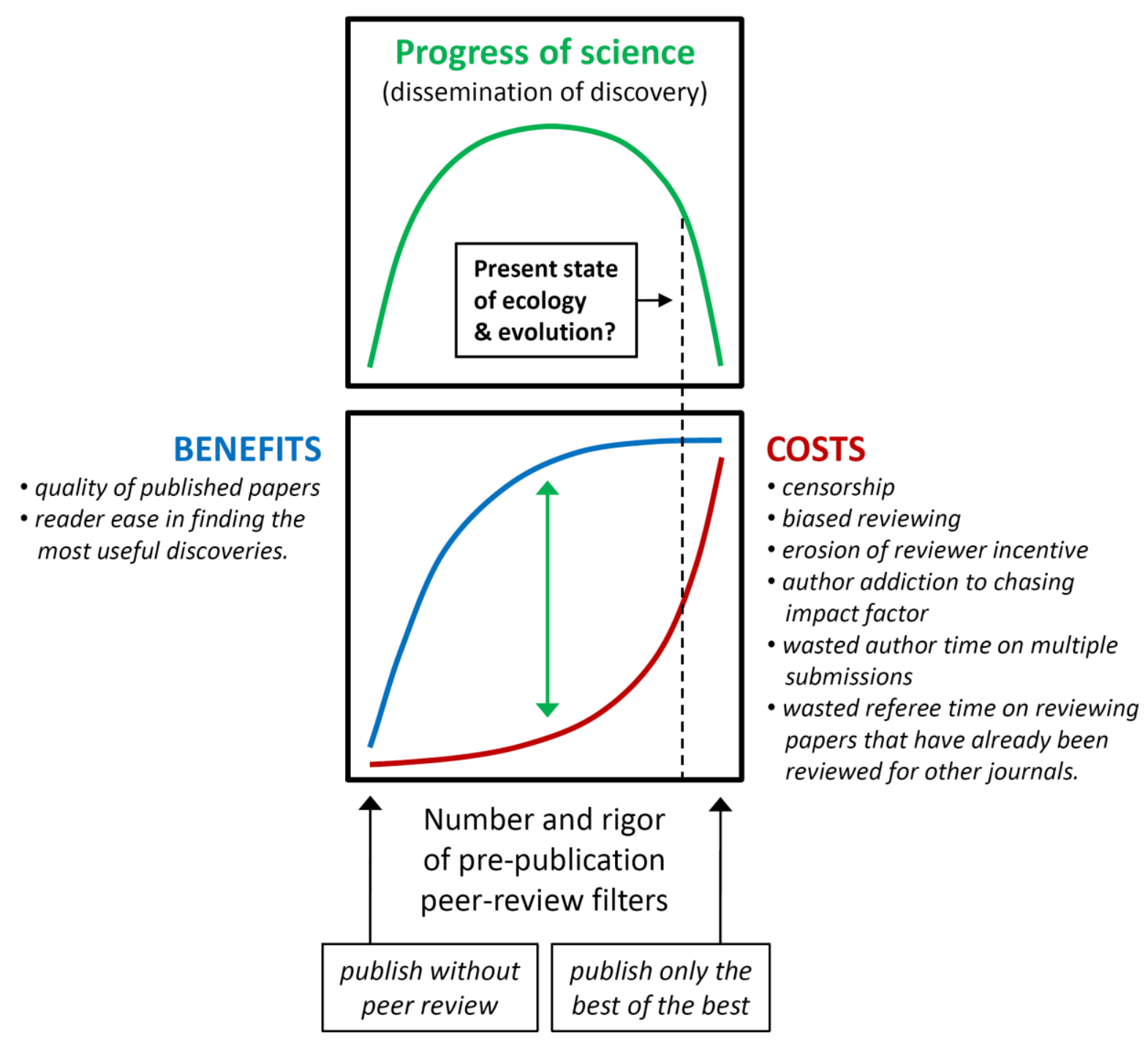

Figure 1. Optimality model for peer-review filters and the progress of science (see text).

and their editors may get elevated prestige-or they may just go through the motions of protecting elitist traditions that they think is their job to protect because they have never known any alternative to chasing impact factor. This addiction to impact factor elitism is so powerful that many editors routinely justify rejection of even high quality papers based on the absurd claim of limited space (or limited budget) for printed pages within paper issues of the journal-despite knowing that paper issues are now redundant. Researchers and students alike, virtually all now read from-and libraries are increasingly subscribing to-only electronic (e.g. pdf format) versions of academic journals.

Editors therefore, are easily empowered to exercise gate-keeping elitism-while conveniently not having to 'look' like elitists - because editors, like reviewers, can also hide. Editors can hide behind two archaic and unnecessary, yet still widely-practiced traditions: paper publication with its attendant competition for limited physical printing space; and anonymous reviewing, where reviewers are given free license to exercise bias without any accountability, because only editors know who they are, and the reviews in most cases are never seen by anyone other than the editor and the author. Importantly, negative reviews (hidden from public view) from aggressive anonymous reviewers (with bias that can be easily and cleverly disguised) provide a valuable tool for publishers and editors committed to gate-keeping elitism: they provide ostensibly legitimate excuses for rejecting very good manuscripts in order to 
ensure that only the 'best of the best' are published in their journals, thus guarding and inflating the sacred journal impact factor.

Ironically then, by previously rejecting a researcher's good paper, an editor creates a jaded reviewer, fashioned with retaliatory reviewing bias contrived to provide exactly what the editor needs: more rejection recommendations of good papers submitted by other authors. Such rejections commonly include no opportunity for the author to even respond to the anonymous reviewer's criticism. Even if the author manages to attract the editor's attention with an appeal, it is often answered with a standard justification based on a peculiar mix of apparent empathy and righteousnesssomething like: "We know this decision must be disappointing but it is important to appreciate that we receive many more high quality submissions than we are able to publish; the intense competition for limited page space within our printed paper issues generally means that we can publish only the best of the best." Hiding behind these traditions enables editors to avoid communicating honestly about why they reject papers that are often equivalent in quality to those already published in their journals. All of this means that decisions about manuscript acceptance for publication from many editors are based not primarily on a dedication to the progress of science; they are instead motivated largely by a fear of floundering in their competition with other journals (and other editors) for impact factor superiority. Commonly, these editors will privately admit that they don't want to risk diluting their journal impact factor by accepting papers that may not capture enough citations to maintain it.

From the beginning of their careers, researchers become so indoctrinated by this now rampant culture of elitism that they seem generally blind to the corruption that it represents, and the crippling impediments that it imposes to the progress of science. It forces authorsespecially young researchers scrambling to build a reputation, compete for grants, and earn tenure and promotion-to relentlessly chase impact factor. This addiction results in wasted time by disillusioned authors submitting the same paper multiple times as they frantically 'ratchet down' the impact factor 'ladder', and wasted time by weary referees reviewing papers that have already been reviewed for other (often several) journals-all competing with each other for impact factor status. Exasperation for some researchers leads to burn-out or academic misconduct, which when exposed results in lost public confidence in the integrity of science and the value of funding it.

Exasperation also generates a run-away decline in reviewer incentive. At best this forces editors to search harder and longer to find willing reviewers. And at worst it generates a segregated class system supported by strong positive feedback, where elitism breeds more elitism because those who publish in elitist journals also become the main reviewers and editors for those journals. In other words, those that win at the current game become the keepers of the rules, and the rules often involve surreptitiously suppressing innovation that threatens the favoured theories of the elites-again limiting the progress of science.

Others have drawn attention to the above problems with the current peer-review system, but researchers in ecology and evolution have done little to address the costs directly. Are ecology and evolution positioned optimally in Figure 1? Is the dissemination of discovery limited by peer-review filters that are generally too stringent? Or are they too lenient? Readers are encouraged to post their views regarding this online at the IEE website (see commenting option after clicking on the present article title in the Table of Contents). The benefits of rigorous peer review are obvious; it is essential for preventing the proliferation of junk science. But we have very little understanding of the above costs that accrue when peer review is excessively stringent. In my view, based on experience as author, reviewer and editor, these costs currently stifle the progress of research in ecology and evolution. Data are needed to test this hypothesis more directly, but one thing is already certain: science is a mission for discovery, nothing else. It is not a mission for elitism, nor is it an arena for journals to compete with each other for impact factor. But this is precisely what it has become, and the corruption propagates itself like a selfish gene. All of this means that traditional elitist journals are unlikely to take the lead in efforts to correct this problem. Ironically, it is in their best interests to keep it in place and to instill complacency about limitations, false pride in the 'traditional way', and false confidence in the view that, although not perfect, the current system is the best we can do.

But it's not; we can do much better. Instead of being indoctrinated to chase journal impact factor, young researchers could be mentored to chase discovery, and to exercise filters - both in their own research practice and in reviewing the research of their peers - that are optimal for the dissemination of discovery (Figure 1). This requires that researchers embrace a revised culture for merit evaluation, where prestige from one's work is something that is earned honestly by metrics that quantify the real impact of one's contributions-author impact factor - not by the impact factor of the journals in which they manage to get published. Author status (e.g. for an ecologist) could be measured using indices (e.g. sponsored by institutions such as Google scholar, or Microsoft Academic Search) that represent the impact of anyone's and everyone's contributions (not just the most 'highly cited') within an entire discipline-e.g. based on citation rate rankings for one's articles published within all journals categorized as 
'ecology'. These could usefully be weighted according to metrics of career stage and research grant size.

Traditional journals - when they were the only game in town-played a central role in the dissemination of discovery. And indicators of journal status, such as impact factor, provided convenient sign posts that could be trusted with some success in pointing readers to important research contributions. But these journals and their impact factors are no longer needed to provide these services for researchers. It is time to give them up, not just because they are products of outdated technology, but even more importantly because many of them - in ecology and evolution at least - have infected science with a cancer that has grown so slowly we have barely noticed it, and it has morphed now into a pandemic culture of elitism that is choking the progress of our science. Researchers have for too long been held captive in the grips of publishers and their dutiful editors who have managed to turn peer review into a tool for the dissemination of impact factor elitism, and for competing with other publishers, journals and editors for the status and profits that impact factor represents.

Thankfully, researchers are now empowered. The digital/internet age has given them the tools and the options for dispensing with profit-based publishers altogether (although they have been surprisingly slow to take full advantage of this freedom). Researchers can now organize themselves to publish their peer-reviewed work electronically (no paper required) in online journals - like IEE - that they control, easily supported on a modest budget needed only for nominal cost recovery (no profit generation required). This in turn releases another major limitation on the current progress of science because public funds and grant budget allocations that now end up in the bank accounts of profit-based publishers can instead be available to support the work of frontline researchers directly.

Researchers no longer need traditional publishers to be their arbiters of quality. They are now equipped to take back control of peer review, and return it to its rightful role as a tool for the dissemination of discovery, where the published product can be openly peerreviewed and openly accessible to everyone, with selfregulating public transparency and accountability, plus easy facilitation for additional post-publication peer review (comment posting) online. The IEE model exemplifies all of these features. Readers also no longer need journal impact factor ratings as a searching tool for identifying particularly meritorious articles within their fields of interest. In addition to evaluations from blogstyle online comment postings at journal websites, readers can also now find the top rated discoveries by consulting professional post-publication peer-reviewing services such as Faculty of 1000. These rating services will become even more comprehensive, more accurate, more efficient, faster and more accessible as online technologies continue to evolve and co-evolve with peer-review culture. The growing market for proofreading, grammar and style consultation services can also be expected soon to make it easy and affordable for virtually all researchers-regardless of their linguistic background or writing prowess - to have equal opportunity to report and submit their important discoveries within high quality manuscripts that can be easily recommended for publication because they will have already been professionally edited.

Old habits die hard, especially when fueled by addiction. But the evolution of new cultural norms can be guided by ethical imperatives. The popularity of gasguzzling urban Hummers fell dramatically in recent years, not because they were no longer available, and not because they no longer advertised status for their owners-but in part because of a shift in collective consumer conscience; urban Hummers were wrong. Concerned citizens were successful in raising awareness that they represented symbols of unacceptable costs to the environment. Likewise, elitist journals - those that censor the publication of good discoveries by entrapping researchers into believing that there is glorification in the employment of Draconian peer-review filtersimpose unacceptable costs on the progress of science. Established researchers - those who already have reputation and tenure, and so nothing to prove and nothing to lose-have the greatest opportunity and obligation to show leadership in breaking free from the contagious and destructive addiction to journal impact factor. Let's work together in helping to transform peer review into a constructive culture based on the primacy of discovery over elitism. All we need to do is get it done. 